Cinémas

Revue d'études cinématographiques

Journal of Film Studies

\title{
Pour une éthique cinématographique
}

\section{Paul Warren}

Volume 4, numéro 3, printemps 1994

Questions sur l'éthique au cinéma

URI : https://id.erudit.org/iderudit/1001035ar

DOI : https://doi.org/10.7202/1001035ar

Aller au sommaire du numéro

\section{Éditeur(s)}

Cinémas

\section{ISSN}

1181-6945 (imprimé)

1705-6500 (numérique)

Découvrir la revue

Citer cet article

Warren, P. (1994). Pour une éthique cinématographique. Cinémas, 4(3), 25-42. https://doi.org/10.7202/1001035ar

\section{Résumé de l'article}

Cet article montre que la violence exacerbée sur les écrans de nos salles obscures vient, essentiellement, de ce que les images cinématographiques commerciales sont devenues des représentations stéréotypées du réel, faisant fonctionner les codes de fascination, entraînant le « choc de la violence figurative du représenté » et produisant « un état de rêve induit chez le spectateur » (Deleuze). Dès lors et inversement, quand il arrive que les images s'étoffent et se nourrissent de symboles pat le travail de l'artiste-cinéaste, la violence n'amène plus nos mains à couvrir nos regards apeurés... Ainsi, contrairement à des oeuvres qui expriment la pensée complexe, comme Paysages dans le brouillard d'Angelopoulos (1989), Jurassic Park de Spielberg (1993) est le paradigme parfait de l'oeuvre close et de l'entreprise immorale. 


\section{Pour une éthique cinématographique}

\section{Paul Warren}

\section{RÉSUMÉ}

Cet article montre que la violence exacerbée sur les écrans de nos salles obscures vient, essentiellement, de ce que les images cinématographiques commerciales sont devenues des représentations stéréotypées du réel, faisant fonctionner les codes de fascination, entraînant le "choc de la violence figurative du représenté" et produisant "un état de rêve induit chez le spectateur" (Deleuze). Dès lors et inversement, quand il arrive que les images s'étoffent et se nourrissent de symboles par le travail de l'artiste-cinéaste, la violence n'amène plus nos mains à couvrir nos regards apeurés... Ainsi, contrairement à des œuvres qui expriment la pensée complexe, comme Paysages dans le brouillard d'Angelopoulos (1989), Jurassic Park de Spielberg (1993) est le paradigme parfait de l'œuvre close et de l'entreprise immorale.

\section{ABSTRACT}

This article shows that the extreme violence that flashes across the screens of our darkened movie theatres results essentially from the fact that the images of commercial cinema have become stereotyped representations of the real that activate codes of fascination and bring in their wake the "shock of the figurative violence of the represented" and produce "a state of induced dreaming in the spectator" (Deleuze). On the other hand, when images are more densely elaborated and incorporate symbols introduced by the artist-filmmaker, the violence no longer leads us to cover our eyes with our hands... Thus, contrary to works that express complexity of thought like Paysages dans le brouillard 
by Angelopoulos (1989), Spielberg's Jurassic Park (1993) offers a perfect paradigm case of a closed work and an immoral undertaking.

Mais ces créatures terrifiantes ne terrifient pas. On voit tout de suite qu'il s'agit d'art et l'art comme on le sait est inoffensif.

Monique Proulx, Homme invisible à la fenêtre

Je propose de partir d'une réflexion de Régis Debray dans son ouvrage récent Vie et mort de limage: une histoire du regard en Occident ${ }^{1}$. En fait, il s'agit de l'avant-dernier paragraphe. Un paragraphe qui est mis en exergue (le seul de tout le livre) : il est séparé de ce qui précède et de ce qui suit par un astérisque. Donc, une pensée que l'auteur juge d'une importance exceptionnelle. Je cite :
[...] une simple question au prochain millénaire : com- ment bien voir autour de soi sans admettre, à côté, en dessous et au-dessus, des "choses invisibles" ? Pas forcé- ment des anges ni des corps astraux. Des réalités idéa- les, mythes ou concepts, généralités ou universaux, im- matérialités ou symboles qui n'auront jamais de traductions visuelles possibles, fussent-elles visuelles dans un cyberspace. Comment peut-il y avoir un ici sans un ailleurs, un maintenant sans un hier et un demain, un toujours sans un jamais?

Ces quelques lignes constituent la morale du livre de Debray. Il faut, de toute urgence, conclut-il - peut-être bien, d'une certaine façon, et dans certains cas —, redécouvrir l'invisible. Puisque les images (celles de la vidéosphère singulièrement), à force de déferler sur nous, en sont arrivées à nous faire croire que tout a été révélé et que n'existe plus que ce qui est vu: "Magie et image ont mêmes lettres et c'est justice. S.O.S. image, S.O.S. magie. Il n'y a qu'un dogme en magie, écrit Eliphas Lévi, et le voici : le visible est la manifestation de l'invisible" (Debray, p. 31). 
Régis Debray rejoint Gaston Fernandez Carrera, qui prend l'image pelliculaire à son origine photographique. Carrera va loin et il inquiète. Il affirme que l'image produite par l'appareil caméra, en réduisant le monde sensible à sa deuxième dimension, reflète l'impossibilité d'affronter la totalité du réel. Il fait la démonstration que l'image photographique, en nous donnant à voir, de plus en plus et de mieux en mieux, les puncta de la surface segmentée du monde, en nous permettant de posséder de l'objet la plus grande proximité possible (p. 17), "répond à une stratégie inconsciente, tel un tropisme menant l'Occident vers son but: se passer d'une réalité dont il n'a plus que faire, tant il est vrai que la réalité persiste dans son intolérable" (note de l'éditeur, en quatrième de couverture). Elle est intolérable, la réalité, au dire de Carrera, parce qu'elle est une "totalité» (p. 22), parce qu'elle est de l'ordre de la "dispersion" (p. 20) et de l'«imperceptible» (p. 39). On lui préfere alors les substituts superficiels et rassurants de la photographie.

Il ne faut pas se méprendre, ce n'est pas l'image en soi que récusent et condamnent Debray et Carrera '. C'est l'image instantanée, c'est le cliché cinématographique. En effet, l'on constate que Debray s'en prend au visuel de la vidéosphère, nominaliste et dénotatif, qui s'est vidé de la richesse polyvalente de l'image iconographique, longuement, nuitamment travaillée par l'artiste poète et mystique (celle que recherche Tarkovski dans ses films); un visuel, en définitive, de la même facture que le cliché photographique analysé par Carrera qui immobilise et, par conséquent, anéantit la vie réelle.

Je veux montrer dans cet article que la violence exacerbée sur les écrans de nos salles obscures vient, essentiellement, de ce que les images cinématographiques commerciales sont devenues des représentations stéréotypées du réel, faisant fonctionner les codes de fascination, entraînant le "choc de la violence figurative du représenté " (Deleuze, 1985, p. 204) et produisant "un état de rêve induit chez le spectateur" (p. 319). Dès lors et inversement, quand il arrive que les images s'étoffent et se nourrissent de symboles ${ }^{2}$ par le travail de l'artiste-cinéaste, la violence n'amène plus nos mains à couvrir nos regards apeurés, mais sécrète "un mouvement développant ses vibrations dans la séquence mouvante qui monte en nous" (p. 204). 
Mais avant de m'embarquer dans l'analyse filmique, je veux surplomber et éclairer mon propos par la réflexion d'Edgar Morin sur la complexité. Morin propose une éthique pour l'an 2000, qui répond aussi bien aux interrogations de Régis Debray que de Gaston Fernandez Carrera. Nous sommes acculés, nous dit Morin, à penser et à œuvrer de manière de plus en plus complexe, afin de combattre "la pathologie moderne de l'esprit [qui] dans l'hypersimplification rend aveugle à la complexité du réel " (1990, p. 23). Il est évident, par exemple, que Spielberg, en hypersimplifiant bêtes et gens dans son Jurassic Park par, entre autres choses, le nettoyage à l'os du roman de Michael Crichton $^{3}$, a plongé dans l'obscurantisme les spectateurs de la salle obscure, en les rendant aveugles à la complexité des dinosaures et, forcément (nous le verrons plus loin), à la complexité des réactions humaines auxquelles se substituent les réactions primaires des personnages écraniques. Il n'est pas moins évident que la violence du film est le résultat direct de l'hypersimplification de la réalité.

Il importe de rappeler que les premiers écrits de Morin, dans les années cinquante, sont axés, en bonne partie, sur le cinéma. Dans son ouvrage célèbre, Le Cinéma ou l'homme imaginaire, Morin amorce sa réflexion en proposant un parallèle entre le cinéma et l'avion. Il s'étonne que le cinéma, inventé en même temps que l'avion et perçu au départ comme étant un instrument scientifique devant aider à la révélation du monde, soit devenu un médium de rêve; exactement à l'inverse de l'avion qui, lui, devait concrétiser les rêves les plus fous de l'humanité et qui est devenu une machine de commerce et de guerre ${ }^{4}$.

Il semble que Morin, qui était fasciné par la pensée complexe, ait voulu retrouver la réalité (à la fois "sujet" et "objet»), le substrat du cinéma infiniment plus riche que ce qu'en donnent à voir et à entendre les images écraniques dominantes. $\mathrm{Ne}$ retrouverait-il pas ainsi la poussée poétique de Vertov et d'Eisenstein pour qui les caméras et les magnétophones de cinéma, de par leur asservissement à la communication mercantile, s'étaient rendus "aveugles à la complexité du réel " et qui, audelà de l'idéologie du matérialisme dialectique, inventaient des techniques originales (l'attraction, l'intervalle) pour libérer les 
images? Il est clair que Morin se situe dans la trajectoire des hommes de science et des artistes qui se sont faits les chantres de la réalité. Il y a dans ses textes scientifiques des accents qui le rapprochent de Victor Chlovski, de Pierre Teilhard de Chardin, d'Umberto Eco, d'Alain Robbe-Grillet, de Jean-Luc Godard. Il est clair également que l'infinie complexité qu'il intuitionne dans la réalité rend, du coup, dérisoires les images clôturées par le champ-contrechamp qui prévalent dans l'industrie cinématographique.

Morin note que le système ouvert, une découverte révolutionnaire en cybernétique, n'a pas su ébranler la théorie du système fermé qui régit l'Occident depuis Descartes, où "toutes choses sont considérées comme des entités closes" (1990, p. 31). Le même type de discours a été tenu par Robbe-Grillet, à l'Université de Los Angeles (U.C.L.A.), il y a une quinzaine d'années. Il venait de projeter son film L'Éden et après (1991) devant plusieurs centaines d'étudiants qui, manifestement, rejetaient l'œuvre difficile, multiforme et pleine de métaphores du cinéaste français. Après quelques questions carrément hostiles, un commentaire d'étudiant a reçu les applaudissements de la salle: " Pourquoi ne faites-vous pas des films comme Truffaut que nous comprenons et que nous aimons?" Excédé, Robbe-Grillet a répondu que Truffaut, comme la majorité des cinéastes français et américains, construisait ses films à partir de structures narratives qui nous ramenaient au XIX siècle. La réaction de la salle, il fallait s'y attendre, a été la rigolade devant cette impertinence bien française.

Et pourtant, Robbe-Grillet ne faisait qu'appliquer au septième art la réalité (non pas la théorie) du "système ouvert", point de départ et centre vivant de la réflexion d'Edgar Morin. $\mathrm{Ce}$ "système ouvert" est bien celui qui "ouvre l'œuvre" pour Umberto Eco. C'est le même "système" qui entraîne Shakespeare à faire s'agiter la mer et gronder le tonnerre dans la lamentation du roi Lear; Munch à faire hurler le ciel dans Le Cri; Brecht à découvrir le vice caché de mère Courage en lui faisant lâcher la main de son enfant pour mordre dans l'écu que lui présente le gendarme recruteur; Joyce à décrire, dans Ulysse, le foisonnement entrecroisé du monologue intérieur de son héros en 
lui faisant acheter trois saucisses chez l'épicier du coin; Faulkner à lier, dans Les Palmiers sauvages, les passions humaines à la débâcle ensauvagée du Mississippi. C'est encore le "système ouvert " qui amène Flaherty à transfigurer en lutte contre la nature la besogne ponctuelle de Nanook aux prises avec son phoque; Eisenstein à célébrer la révolution des masses par la levée du pont sur la Néva qui sépare les deux rives du monde, le bon grain de l'ivraie, le cheval blanc de son carrosse et la femme de sa chevelure blonde. C'est toujours le "système ouvert" qui pousse Vertov, dans Enthousiasme, à monter le plan de l'ivrogne qui lève le coude avec le plan de la bigote qui baisse le bras pour se signer; Godard à relier cosmiquement le ventre rond de $\mathrm{Ma}$ rie à la plénitude de la lune; Fellini à couper le match qui commence entre les deux amazones des Clowns pour éviter que le conflit qu'il veut social ne se dégrade dans le corps à corps individuel; et Robbe-Grillet, justement, à présenter dans un plan unique de L'Éden et après, une femme nue enchaînée et se tordant de douleur cependant que son tortionnaire frappe à coups redoublés sur un matelas.

Se distancer pour créer. Cinématographiquement, repousser le plus loin possible les unes des autres les images qui, par habitude, "prématurément", comme dit Godard, s'accouplent bêtement en champ-contrechamp. Donner du champ à la métaphore. Surtout, s'organiser structurellement pour que «[...] la réalité - la flamme d'une bougie, un tourbillon, une cellule (et quelque image que ce soit du réel) — soit autant dans le lien que dans la distinction entre le système ouvert et son environnement" (Morin, 1990, p. $32^{5}$ ). Pour la bonne raison que, "logiquement, le système ne peut être compris qu'en incluant en lui l'environnement, qui lui est à la fois intime et étranger et fait partie de lui-même tout en lui étant extérieur " (p. 32).

À l'évidence, pour Morin, le "système ouvert " est incontournable. Il est l'outil essentiel de "la pluralité complexe et anthroposociale de l'éthique" (Morin, 1981, p. 296), laquelle doit régir désormais notre pensée et nos œuvres si nous voulons "sortir du XXe siècle». Or, il s'en faut de beaucoup qu'il en soit ainsi. C'est toujours le "système clos" qui sévit dans notre pensée politique et artistique. «La stratégie manichéenne sans cesse redéfi- 
nit le bien par rapport au mal» (p. 299) et évacue constamment la complexité du comportement humain. La pensée qui détermine les œuvres contemporaines à succès est une "[...] pensée simplifiante, disjonctive, antagoniste, qui isole ce qu'elle sépare, et occulte tout ce qui relie, interagit, interfere" (Morin, 1990, p. 11). C'est " [...] une pensée mutilante qui conduit nécessairement à des actions mutilantes" (p. 23) et qui " [...] entraîne une vision du monde classificationnelle, réductionniste, une causalité unilinéaire ${ }^{6} "$. Cette pensée est claire et définitive. Elle donne une impression de totalité qui plonge l'esprit dans la "non-vérité ", comme l'écrit Adorno ${ }^{7}$. Mythique, elle est le contraire de la pensée complexe qui ressortit à la réalité et qui "doit affronter le fouillis (le jeu infini des interrétroactions), la solidarité des phénomènes entre eux, le brouillard, l'incertitude, la contradiction" (p. 22).

"Brouillard". Ce mot me ramène à la mémoire le merveilleux film d'Angelopoulos, Paysages dans le brouillard (1989). Il y a, dans cette œuvre majeure du réalisateur grec, une séquence en particulier qui exprime remarquablement ce que vient de dire Morin sur la pensée complexe et qu'il importe d'analyser un peu. Il s'agit de la séquence du viol de la jeune fille. Deux plans pour cinq minutes d'écran, deux mouvements de caméra et trois mots prononcés. Et un foisonnement de pensées et de monologues intérieurs dans la salle qui émerge de son obscurantisme. Voyons un peu. La caméra prend de face, en plan moyen, le camion qui s'arrête sur le bord de l'autoroute. Notons que le plan moyen permet une saisie phénoménologique du réel, à michemin entre la trop grande subjectivité du gros plan et la trop grande objectivité du plan d'ensemble. À travers la vitre, nous reconnaissons les passagers: les deux personnages principaux, la gamine de 12 ans et son petit frère de 5 ou 6 ans qui s'est endormi près d'elle, le camionneur qui les a embarqués une séquence auparavant. Le camionneur dit: "Je vais roupiller." Il ouvre la portière et se dirige vers l'arrière de son camion. La caméra opère alors un très lent travelling latéral vers la gauche et s'immobilise sur le côté du passager du véhicule, laissant s'ouvrir au loin une partie de l'autoroute. Après un moment assez long où nous entendons le bruit du trafic sur l'autoroute (bruit qui 
va persister tout au long de la séquence), le camionneur apparaît. Il a changé d'idée. Il finit de boire son café, jette le verre en polystyrène sur le bord de la route et, après un temps d'hésitation, ouvre la porte du camion: "Viens!", dit-il à la petite fille. Celle-ci refuse d'abord puis descend, passe devant et se met à fuir en direction opposée à la caméra. L’homme la poursuit, l'attrape en profondeur de champ (la caméra ne bouge pas) et la traîne malgré sa résistance vers la cage du camion.

Le deuxième plan de la séquence s'installe ici. La trajectoire de la caméra s'inverse. Nous voyons l'arrière du camion qui est fermé par une toile. Nous sommes toujours en plan moyen, mais qui laisse, cette fois, s'ouvrir sur la gauche une partie de l'autoroute. Nous observons, d'une certaine façon, le contrechamp du champ précédent. Nous observons, nous ne sommes pas pris au piège du champ-contrechamp producteur de notre vision comme c'est le cas dans le cinéma commercial. Nous voyons le camionneur soulever la toile et pousser la petite fille à l'intérieur du camion. Puis, nous attendons, toujours à la même distance respectueuse, sans rien voir que cette toile sombre et souillée devant nous et le va-et-vient des véhicules qui filent bruyamment sur notre gauche. À un moment donné, nous entendons une musique venant d'une voiture qui se range sur la route, à une centaine de pieds devant le camion, donc en grande profondeur de champ. Une seconde voiture s'amène et s'immobilise à la hauteur de la première. Un mystérieux échange d'objets se trafique et les deux voitures reprennent la route. Alors on entend en off la voix du petit garçon crier à plusieurs reprises le nom de sa sœur. Sans cesser d'appeler, il débouche devant nous, à droite du camion, le contourne, le longe sur la gauche jusqu'à la cabine du chauffeur, revient vers l'arrière et disparaît au loin. Alors, réapparaît le camionneur. Il soulève la toile, en regardant à droite et à gauche avec inquiétude, met pied à terre, va vers la cabine, revient, s'appuie sur le côté du camion, porte la main à sa bouche, revient sur ses pas, ouvre la portière et disparaît à notre vue. On ne le reverra plus de tout le film. Un moment d'attente encore et la jeune fille refait surface en même temps que la caméra se rapproche en travelling avant. L'on voit ses jambes d'abord qui sortent sous la toile, puis le 
reste du corps. Elle reste assise en silence, levant à la hauteur de ses yeux sa main ensanglantée. Fondu au noir.

Constamment dans cette séquence, l'image transpire au-delà du donné factuel. Pour la bonne raison que jamais l'image ne se ferme sur elle-même, réussissant à fonctionner comme un signe qui dit (ce qui pourtant est le propre du mot) tout en ne cessant de nous imposer sa présence à l'écran, ainsi que l'ont écrit, à leur manière et l'un aussi bien que l'autre, les poètes Pasolini et Pingaud. Ce qui se passe alors, c'est que le spectateur, occupé par la multiplicité du sens qu'on lui donne à gérer, amené à la prise de conscience sur le viol (il faut rappeler que la prise de conscience ne se produit que dans le rapport avec autre que soi), ne peut penser, un seul instant, se lancer, linéairement, par héros interposé, à la poursuite du salaud qui a violé la petite fille.

C'est malheureusement la pensée systématiquement fermée qui sous-tend les œuvres filmiques les plus populaires, les seules que verront l'immense majorité des spectateurs du grand comme du petit écran. Il me paraît de plus en plus que le désarroi des jeunes dont on parle savamment dans les colloques internationaux est dû, pour une grande part, à la contradiction qu'ils ressentent confusément, à la frange de leur plaisir, entre les films hypersimplifiés qu'il leur est donné de voir et d'entendre et la réalité hypercomplexe à laquelle ils doivent se confronter pour vivre. Ils sentent qu'ils sont devant une absence et un leurre, et non pas sollicités par des synthèses d'images et de sons qui ouvrent leur réalité en la transfigurant. Godard exprime cela dans Masculin/Féminin, un de ses nombreux films sur la déperdition de la jeunesse parisienne. Dans une séquence qui se passe dans une salle de cinéma, le personnage principal, Paul (Jean-Pierre Léaud), devant l'écran où se déroulent linéairement des images de violence sexuelle, se dit par-devers lui-même, le visage empreint d'une grande tristesse: "Ce n'est pas le film que j'aurais aimé voir."

Avec leur Jurassic Park, Spielberg et $c^{\text {ie }}$ ont réussi le plus gros succès écranique non seulement de l'été 1993, mais également de toute l'histoire de l' "hollywoodisme". Or, ce film est le paradigme parfait de l'œuvre close. Idéologiquement et structurellement (mieux, idéologiquement parce que structurellement), 
tout y est clair, péremptoire, définitif, unilinéaire; rien d' "invisible en dessous, au-dessus" du cadre planifié où l'image est emprisonnée, de telle manière, en vue d'un tel emboîtement "montagiste" obligatoire qu'elle ne saurait se dire au-delà de la figuration immédiate du représenté. Et pour s'assurer que le spectateur de la salle obscure ne puisse voir et entendre que ce lumineux-là, Spielberg, à sa manière de rhétoricien professionnel de la manipulation, opère une "prise de regard" (Zimmer, p. 66) en orientant le tir de sa caméra, sa "caméra-tir", selon l'expression de Virilio (p. 66), selon une trajectoire dont il n'est plus possible de déroger.

Avant que ne commence l'aventure dans le parc à dinosaures, le paléontologue, qui va se révéler vite le héros de l'histoire, interrompt ses fouilles dans le désert du Montana pour sermonner un garçonnet d'une douzaine d'années, à l'œil dubitatif et rieur, qui a fait l'erreur (cinématographique) de regarder ailleurs au moment où l'on a dégagé des sables le fossile d'un vélocipator; pire, il s'est risqué à comparer le dinosaure à une grosse volaille inoffensive. Le scientifique ${ }^{8}$ lui sert alors un discours terrifiant. Il s'approche du jeune délinquant, le contourne et le circonvient en le fixant intensément pour mieux le cibler et, les yeux dans les yeux, le verbe et le geste tranchants, lui explique, en forçant sur le détail saisissant, la férocité de la bête. Il met un terme à son opération d'envoûtement en lâchant: "Quand il te dévore les entrailles, tu es vivant. " Immédiatement après l'horreur de cette dernière phrase vient se loger, au centre géographique de l'écran lumineux, le dernier plan de la séquence: un gros plangeôle du gamin dont les yeux tentent encore, un moment, de se dérober à l'emprise de la "caméra-tir" et qui, au bout de l'enserrement immobile de l'image, fixent sans ciller le hors-champ d'où vont surgir les monstres ${ }^{2}$. À ce stade du film, l'opération du "dressage de l'œil» est solidement engagée. Dans les trois salles de cinéma où j'ai observé le phénomène, les jeunes spectateurs - ceux, notamment, qui étaient venus en bande et qui, jusque-là, rigolaient encore de temps en temps entre eux, en tournant la tête à droite et à gauche - s'étaient assagis et regardaient droit devant eux. Les œillères sont installées qui séparent les enfants les uns des autres et les orientent tous dans une 
trajectoire unique vers et dans les images unilinéaires qui vont se monter pour eux. C'est le syndrome de la caverne de Platon.

Mais le produit hollywoodien s'adresse à tout le monde. Les spectateurs adultes de Jurassic Park doivent également subir un dressage du regard, lequel intensifiera une fois encore et par osmose celui des jeunes spectateurs. C'est le couple le plus empathique de la distribution, le plus crédible aussi, le paléontologue et sa collaboratrice la paléobotaniste, qui a l'insigne privilège de voir, le premier, les dinosaures du parc. Spielberg monte (dans le sens d'organiser et de graduer) leur vision(nement) en utilisant, au maximum de son efficacité, la structure éminemment "identificatoire" du plan de réaction ${ }^{10}$. Précisons, au meilleur de notre mémoire, la manière dont il amène les deux scientifiques à fixer, l'un et l'autre, l'un après l'autre, le hors-champ et, partant, à entraîner la salle dans leur trajectoire réactionnelle. La jeep dans laquelle a pris place le couple d'experts en dinosaures s'arrête au milieu d'un plan d'ensemble du parc. La caméra prend le véhicule de face en plan moyen afin de capter l'opération des regardants sur le regardé. C'est le regard du paléontologue qui opère le premier. Qui mieux qu'un maniaque des animaux préhistoriques, n'ayant jamais eu jusqu'ici autre chose à observer sous son grattoir que des fragments d'os fossilisés, pourrait connaître la béatitude à la vue de dinosaures vivants? La caméra, qui le fixe depuis un moment, se rapproche au fur et à mesure que son regard passe de l'indifférence à l'envoûtement. Le comportement du paléontologue est longuement travaillé. Presque au ralenti. Chaussé de verres fumés noirs qui masquent ses yeux et annulent pour nous l'orientation de son regard, il lève la tête vers nous. Soudainement, il s'immobilise. Il voit! (Nous savons qu'il a vu). Lentement, il lève le bras et enlève son chapeau (pas ses verres, pas encore). Tout aussi lentement, il se redresse et se met debout dans la jeep. C'est alors qu'il libère ses yeux des deux cercles noirs qui nous empêchaient de voir son regard que nous sentions déjà, depuis un moment, fasciné et qui nous apparaît maintenant exorbité par le ravissement. Mais nous, spectateurs, nous ne voyons pas encore ce que voit le paléontologue, ce qu'il n'a pas assez d'yeux pour voir. Ce hors-champ intensément anticipé par la vision privilégiée de notre délégué 
écranique ne devient pas tout de suite notre champ de vision. Spielberg ne prend pas de chances parce qu'il n'a pas un sou à perdre. Dans son entreprise d'arrimage de la salle à l'écran, pour être bien sûr de ferrer le spectateur, il donne une alliée au paléontologue-regardant. Celui-ci, sans quitter le hors-champ qu'il dévore des yeux, cherche à attirer l'attention de sa collègue la paléobotaniste. Il lui touche la tête de la main. Elle regarde alors dans la même direction que lui. Elle porte elle aussi des verres noirs qu'elle enlève, sidérée, en se levant debout dans la jeep, aux côtés de son compagnon. Quatre paires d'yeux qui, dans la stupéfaction, ne cillent plus, retrouvant l'aperture de la "caméra-tir".

Les spectateurs qui, dans l'obscurité de la salle, écarquillaient déjà les yeux à la mesure du regard exorbité des deux spécialistes en dinosaures sont mûrs pour voir enfin. Mais pour que leur arrimage à l'écran se fasse dans l'exacte trajectoire de leurs délégués écraniques, ceux-ci sont introduits dans le plan d'ensemble, au premier plan du plan d'ensemble, aux premières rangées pour ainsi dire, levant les yeux avec eux, pour eux, en même temps qu'eux, vers les gigantesques brontosaures qui se déploient dans la plaine.

Lon pourrait penser qu'il n'y a là rien d'alarmant pour la qualité de l'œuvre et que ce montage, longuement rodé depuis des décennies, de l'instance réactionnelle des regardants écraniques permet, simplement, d'attirer l'attention du spectateur pour une histoire qui, par ailleurs, peut tout à fait se payer le luxe d'être complexe. Ce serait oublier que cette stratégie montagiste, en orientant dans une direction unique le regardant spectatoriel, ne présente à la vue, du même coup et obligatoirement, qu'une facette du regardé. Et cette facette unique, singulièrement éclairée sous le spot du projecteur et dûment ciblée par la caméra-tir, est regardée par le spectateur selon le même degré d'émotion et d'intensité que celui de ses délégués. Impossible pour le spectateur de voir autre chose que ce que voit son délégué ou de le voir autrement qu'il ne le voit. Le couple du parc anticipe et inclut dans son regard le regard du spectateur. Mieux, ce regard-là crée la vision du spectateur, l'orientation de cette vision et jusqu'à son objet. Il s'agit, en termes cinématographiques, d'un 
contrechamp qui est donné au spectateur avant que ne s'installe son champ de vision. En plus clair, si l'on se situe au niveau global du système-cinéma où la salle obscure est le contrechamp (Metz dirait le hors-champ ") de ce champ qu'est l'écran lumineux, l'on peut dire que, avec l'hollywoodisme spielbergien, avant même que ne soit donné au spectateur son champ de vision, il est déjà préfabriqué en douce, dans l'obscurité des salles, à même le comportement réactionnel des personnages. En conséquence, rien de nouveau n'est connu, il n'y a plus qu'une reconnaissance de ce qui était là au départ.

Il n'est que d'examiner un instant deux autres patterns, systématiquement utilisés par Spielberg dans tous ses films et, singulièrement, dans Jurassic Park, l'espace en attente du personnage et le montage parallèle, pour se rendre compte qu'ils ne sont que le prolongement naturel du plan de réaction écranique, destinés à soutenir et à renforcer, dans les scènes d'action narrative intense, l'hypertrophie du regard spectatoriel. L'espace en attente du personnage est cet espace vide où le héros n'est pas encore, qui nous est donné, quelques secondes durant, à nous, spectateurs, et dans lequel nous attendons, sachant sans l'ombre d'un doute qu'il est le seul et unique lieu dans lequel viendra, fatalement, le personnage, emportant avec lui l'émotion et le comportement que nous anticipons d'autant plus sûrement que nous les avons déjà faits nôtres. $\mathrm{Si}$, comme nous l'avons vu, le comportement réactionnel de notre délégué écranique remplit l'écran pour que le regard du spectateur puisse s'installer correctement dans sa mouvance, l'espace vide nous est donné pour que nous le remplissions de la réaction émotionnelle qui est déjà celle du personnage qui va venir nous rejoindre dans un instant. Quant au montage parallèle, qui se présente, comme dit Deleuze, "comme du bacon avec son alternance de gras et de maigre" (1983, p. 50), il est l'exacte extension de l'espace en attente du personnage. De même qu'on aboutit à un establishing shot de l'ordre de la coupe dans le mouvement, si l'on réduit au maximum l'espace qui précède la venue du personnage, ainsi en arrive-t-on au montage parallèle si l'on étire au point de l'élaborer en ligne narrative autonome le temps de représentation de cet espace anticipé. Le spectateur dont le regard est déjà 
domestiqué, "écranisé " si l'on peut dire, alterne alors d'une action narrative à une autre, avec d'autant plus de complaisance que se réalise filmiquement l'alternance cinématographique primordiale salle / écran; deux champs antagonistes (showdown approach) profondément connotés par l'alternance du regardé/ regardant écranique qui reprend de plus belle (parce que la situation est paroxystique) aussi bien dans l'un que dans l'autre; deux champs enfin qui escamotent la coupe entre eux (comme l'œil qui oublie de ciller), pour la bonne raison que le spectateur anticipe leur apparition d'autant plus fortement qu'il a été amené à sécréter la gestuelle et le comportement réactionnel qui s’y déploient.

Ce qu'il faut préciser pour notre propos, c'est que les trois structures ou patterns dont nous venons de parler, plan de réaction, espace en attente du personnage et montage parallèle, ne sont que des variations d'un même système binaire en champcontrechamp, primaire et manichéen, qui ne saurait produire et gérer que des contenus à leur image et leur ressemblance.

Que nous est-il donné à voir et à entendre dans Jurassic Park? Une alternance constante par le biais non moins constant du regardé/ regardant entre, d'une part, l'innocence enfantine et la bonté adulte dans toute leur pureté et, d'autre part, les vices sordides des vilains auxquels fait écho la férocité absolue des bêtes. De ces deux univers antagonistes ne surnagent à la surface de l'écran (n'ont survécu en somme à l'épuration scénaristique de l'œuvre romanesque de Michael Crichton) que des clichés que le spectateur, réduit qu'il est à "l'homme unidimensionnel ", reçoit de plein fouet d'autant qu'il les porte en lui.

Lextrême violence du film se manifeste, de façon particulière, cela va de soi, dans les séquences où il y a mort d'hommes. Joue alors, jusqu'à l'exacerbation, la structure duale conventionnelle, avec à son bord le plan de réaction du regardant pétrifié d'horreur et l'espace anticipé qui s'accentue jusqu'à créer l'alternance. Les victimes sont formellement prises entre les mâchoires du champ-contrechamp. Mais qui sont ces victimes? Quatre individus représentant des connotations catégorielles profondément enfouies dans l'inconscient populaire américain: le lâche, le traître, l'inadapté et l'inadaptable. 
C'est un avocat qui lâche les enfants. Un avocat de corporation (corporation lawyer) véreux, dénué de principes, égoïste et ne s'intéressant qu'au profit, résumant de façon caricaturale l'image négative que se fait de la profession le gros du public. Il quitte en panique la voiture qu'il occupait avec les deux enfants, le frère et la sœur, au moment où le tyrannosaure qui les a repérés fonce sur eux. Il sera dévoré dans une scène punitive, l'une des plus violentes du film, que Spielberg a renforcée en l'alternant brièvement avec son contraire, celle où l'adulte au grand cœur sauve les enfants.

C'est l'informaticien en chef du parc qui trahit, celui qui tient entre ses mains la vie de tous les actants du film. Le réalisateur l'a voulu obèse, glouton et, surtout, excentrique. Déjà, paradoxalement, en dehors du cercle. Dans les trois séquences où il apparaît, il a un comportement expressionniste, étrange et inquiétant, rappelant ces vilains qui circulent dans le filmique américain du western jusqu'à Batman et qui n'arrivent pas à contrôler leurs rires d'hystériques. Il abandonne son poste et se sauve en pleine nuit, après avoir volé les embryons de dinosaures et détraqué le système électronique. Il se fera dévorer dans une séquence en montage parallèle antinomique à suspense, longuement élaborée, qui nous entraîne à désirer sa mort.

Les deux autres victimes des dinosaures ne sont pas des vilains. Le premier, le gardien du parc, un ancien chasseur de brousse, est un homme courageux, mais il a un comportement anachronique: il utilise une carabine contre des bêtes préhistoriques. Le second, un subalterne sympathique, un Noir (le seul du film, car il faut exclure celui, cobaye et anonyme, qui meurt de façon atroce dans le générique) spécialiste des systèmes de sécurité du parc, devient absolument incapable de fonctionner quand l'informaticien n'est plus là pour l'alimenter en données électroniques. Ni l'un ni l'autre ne se rendent coupables de quoi que ce soit. Ils sont simplement dépassés par les événements et leur mort, pour ainsi dire, va de soi. Aussi, si le champ-contrechamp du regardé/ regardant (du chasseur/ chassé) est utilisé pour marquer l'erreur mortelle du gardien, cette structure binaire de base ne se rend jamais jusqu'au montage parallèle qui nous ferait anticiper la mise à mort. Quant au Noir, devenu 
inutile après quelques services rendus, il est carrément évacué du champ des Blancs. C'est dans le hors-champ, dans l'inexistence filmique qu'il meurt.

Tout cela pour dire que les quatre morts de Jurassic Park s'inscrivent sur l'écran, fond et forme inclus, comme des clichés (dans le sens photographique du terme) déclenchant en nous des signaux de repérage automatique. Dans la salle obscure, au fond de notre obscurantisme, se lèvent venant de nos origines les plus lointaines des images ensauvagées qui nous disent, de façon claire, péremptoire, définitive, que les lâches, les traîtres, les inadaptés et les inadaptables (a fortiori s'ils sont Noirs) doivent être punis de mort, de manière plus ou moins violente selon leurs actes, inéluctablement et sans autre forme de procès. Aucune porte de sortie ne nous est offerte. Aucun «in-vu " au-delà du montré, aucun non-dit qui permettrait de loger notre discours intérieur. Nous sommes pris au piège de la figuration immédiate, exclusive et stéréotypée du représenté. C'est pourquoi la violence anonyme et silencieuse des salles obscures est si grande, qui reflète la violence fortement dénommée et vociférée de l'écran.

Le $\mathrm{XXI}^{\mathrm{e}}$ siècle ne pourra se construire que dans la complexité, c'est-à-dire dans la simultanéité d'une multitude infinie de projets et de lignes d'action, à même des "œuvres ouvertes" qui permettent de "bien voir autour de soi, à côté, en dessous et audessus des choses invisibles". C'est pourquoi, des produits comme Jurassic Park deviennent de plus en plus clairement des entreprises immorales. Or, s'il est vrai qu'une éthique de notre temps " $[\ldots]$ ne peut que travailler avec la complexité" (Morin, 1981, p. 297), il devient évident que des produits comme Jurassic Park sont anachroniques et, de par leur envahissement de notre espace écranique, profondément nocifs.

Université Laval

\section{NOTES}

1 L'on pourrait leur adjoindre de nombreux autres penseurs, notamment Walter Benjamin, Susan Sontag, Roland Barthes, Pierre Bourdieu, Gilles Deleuze, Serge Daney, Neil Postman, Paul Virilio... 
2 Je prends ici le terme symboliser dans son sens étymologique grec, sumballein, qui signifie réunir, assembler. Je privilégie la définition qu'en donne Hughes de SaintVictor: "Assemblage de formes visibles pour la démonstration de choses invisibles." Cf. Yvan Illich, Du lisible au visible: sur l'art de lire de Hughes de Saint-Victor (Paris: Cerf, 1991), p. 42.

3 Étrangement, Crichton a collaboré, personnellement, à la scénarisation mercantile de son œuvre romanesque.

4 Le philosophe Paul Virilio (dont la pensée a des affinités avec celle de Debray et de Carrera) ne voit pas les choses selon la même optique. Il découvre avec effroi que l'invention de l'avion et celle du cinéma étaient nécessaires pour faire la guerre totale. Il note que "la caméra va être engagée, dès le début, dans la guerre aérienne, parce que la guerre détruit tout, que les cartes d'état-major ne suffisent plus et qu'il faut absolument une mémoire-cinéma pour s'y retrouver et ramener la preuve de la destruction. La chasse aérienne a réussi à coupler sous le nom de ciné-mitrailleuse l'invention de la photographie à répétition qu'est le cinéma ( 24 images/seconde) et l'invention du fusil à répétition qu'est la mitrailleuse " ( Cinéma-vitesse”, dans Revue d'esthétique, Nouvelle série, $\mathrm{n}^{\circ} 10,1986$, p. 39).

5 La comédienne Anna Prucnal, chantre avertie de Brecht, s'arrête soudain, au paroxysme d'un song, met un genou en terre, baisse la tête et s'immobilise dans une minute de silence. Elle entraîne ainsi le public à une réflexion vertigineuse, lui faisant goûter au symbole dans le sens profond que lui donnait Hughes de Saint-Victor (voir note 2).

6 Morin cite ici M. Maruyana dans "Paradigmatology and its application to crossdisciplinary, cross-professional and cross-cultural communication", Cibernetika, $\mathrm{n}^{\circ} 17$ (1974).

7 Cité par Morin, Introduction à la pensée complexe (Paris: E.S.P., 1990), p. 11.

8 Il est stratégiquement important, en cinéma hollywoodien, que la science vienne accréditer l'histoire. C'est l'habitude de Spielberg de fonctionner ainsi. On se rappelle le discours de l'océanographe sur la sauvagerie du requin blanc (Jaws, 1975), juste avant le départ en mer des trois hommes.

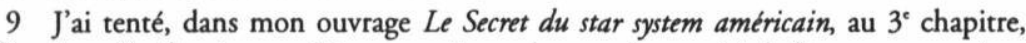
d'analyser l'utilisation par Murnau et Lang du regard terrorisé de l'acteur expressionniste (Montréal : l'Hexagone, 1989).

10 Le reaction shot, comme je tente de le démontrer dans mon ouvrage précédemment cité, a été rodé par Hollywood à partir des années qui marquent le début des grandes manœuvres en vue de la "vedettarisation" des stars. De tous les patterns hollywoodiens, c'est celui qui assure le mieux la vente des vedettes aux spectateurs, le to sell stars to the public qui était la règle d'or des grands studios californiens.

11 Voir Le Signifiant imaginaire (Paris: U.G.E., 1977), p. 77.

\section{OUVRAGES CITÉS}

Carrera, Gaston Fernandez. La Photographie, le néant: digression autour d'une mort occidentale. Paris: P.U.F., 1986.

Debray, Régis. Vie et mort de l'image: une histoire du regard en Occident. Paris: Gallimard, 1992.

Deleuze, Gilles. Cinéma 1. L'Image-mouvement. Paris: Minuit, 1983.

Deleuze, Gilles. Cinéma 2. L'Image-temps. Paris: Minuit, 1985.

Eco, Umberto. L'Euvre ouverte. Paris: Seuil, 1979. 
Morin, Edgar. Introduction à la pensée complexe. Paris: E.S.P., 1990.

Morin, Edgar. Le Cinéma ou l'homme imaginaire. Paris: Minuit, 1978.

Morin, Edgar. Pour sortir du XX' siècle. Paris: Nathan, 1981.

Proulx, Monique. Homme invisible à la fenêtre. Montréal/Paris: Boréal/Seuil, 1993.

Zimmer, Christian. Procès du spectacle. Paris: P.U.F., 1977. 